\title{
Extended Pharmacologic Thromboprophylaxis in Oncologic Liver Surgery is Safe and Effective
}

\author{
Bradford J. Kim, MD, MHS ${ }^{1}$, Ryan W. Day, MD², Catherine H. Davis, MD', Nisha Narula, MD¹, \\ Michael H. Kroll, MD², Ching-Wei D. Tzeng, MD¹, and Thomas A. Aloia, MD' \\ ${ }^{1}$ Department of Surgical Oncology, The University of Texas MD Anderson Cancer Center, \\ Houston, Texas \\ 2Department of Surgery, Mayo Clinic, Phoenix, Arizona \\ ${ }^{3}$ Section of Benign Hematology, Division of Internal Medicine, The University of Texas MD \\ Anderson Cancer Center, Houston, Texas
}

\begin{abstract}
Background-The incidence of venous thromboembolism remains high after liver surgery.

Objective-To evaluate the safety and efficacy of extended pharmacologic thromboprophylaxis in liver surgery for the prevention of venous thromboembolism (VTE).
\end{abstract}

Patient/Methods-From August 2013 to April 2015, 124 patients who underwent liver resection for malignancy were placed on an extended pharmacologic thromboprophylaxis protocol. Intraoperative VTE prophylaxis included Thrombo-Embolic Deterrent hose and sequential compression devices. Once hemostasis was assured following hepatectomy, daily anticoagulant VTE prophylaxis was initiated for the hospitalization. After hospital discharge, the large majority of patients $(114,91.9 \%)$ continued anticoagulant thromboprophylaxis (enoxaparin) to complete a total course of 14 days after minor/minimally invasive (MIS) hepatectomy or 28 days after major hepatectomy or a history of VTE.

Results-The cohort included 39 (31.2\%) major hepatectomies and 38 (31.5\%) MIS approaches. The intraoperative, postoperative and overall transfusion rates were $5.6 \%, 8.1 \%$ and $10.5 \%$ respectively. Pharmacologic thromboprophylaxis was started on postoperative day (POD) 0 for 40 (32.3\%) patients and POD 1 for 84 (67.7\%) patients. During 90-days of follow-up, no postoperative symptomatic DVT or pulmonary embolic events were diagnosed. Standard protocol computed tomography scans of the chest/abdomen/pelvis that were obtained on $112(90.3 \%)$ study

Address all correspondence and reprint requests to: Thomas A. Aloia, MD, University of Texas MD Anderson Cancer Center, Department of Surgical Oncology, 1400 Herman Pressler Drive, Unit 1484, Houston, TX 77030, Phone: (713) 563-0189, Fax: (713) 745-6287, taaloia@mdanderson.org.

\section{Addendum}

T. A. Aloia, B. J. Kim, and R. W. Day contributed to the concept and design of this study. T. A. Aloia, B. J. Kim, C. H. Davis, and N. Narula contributed to data analysis.

All authors contributed to the interpretation of the results.

T. A. Aloia, B. J. Kim, M. H. Kroll, C. D. Tzeng, C. H. Davis, and N. Narula contributed to the writing of the manuscript. All authors contributed to the critical revision of the final manuscript.

Disclosure of Conflict of Interests

The authors state that they have no conflict of interest. 
patients identified no pulmonary emboli, other thoracic, splanchnic, or ileofemoral vein thromboses. Two (1.6\%) patients had minor bleeding events that resolved after discontinuation of enoxaparin, requiring neither blood transfusion nor reoperation. The severe complication rate was $5.6 \%$, with no 90-day mortalities.

Conclusions-These preliminary data suggest that extended pharmacologic thromboprophylaxis for liver surgery patients is safe and effective.

\section{Keywords}

anticoagulants; hepatectomy; morbidity; pulmonary embolism; venous thrombosis

\section{INTRODUCTION}

Despite an increasing magnitude and complexity of hepatectomy, the safety of modern era liver surgery has seen significant improvement.[1-10] However, rates of postoperative venous thromboembolism (VTE) remain significant at $1-5 \%$, consistently doubling the rate associated with colorectal surgery. Data from the American College of Surgeons National Surgical Quality Improvement Project (ACS - NSQIP) database demonstrate a 2.9\% rate of symptomatic postoperative VTE among 5,706 liver resection patients.[11] Subsequent analysis of the NSQIP database revealed that one third of symptomatic post-hepatectomy VTE were diagnosed after hospital discharge.[12] Despite the publication of guidelines, [13] a specific protocol that includes pharmacologic prophylaxis of VTE after hepatectomy has not been unanimously agreed upon, and the role for anticoagulation remains controversial.

Historically, liver surgeons have tended to avoid postoperative VTE chemoprophylaxis due to a perceived risk for perioperative bleeding.[11] The practice of complete avoidance or late initiation of thromboprophylaxis after decline of the International Normalized Ratio (INR) was further supported by an erroneous misconception that postoperative liver insufficiency, particularly common following major and extended hepatectomies, lead to coagulopathy that protected against VTE. More recent data, however, have defined the concept of "rebalanced coagulation" [14] and suggest that patients are usually in a prothrombotic state after hepatectomy.[15,16]

Following a Clinical Practice Guidelines Conference Series focused on Venous Thromboembolism Prophylaxis in Liver Surgery at the Americas Hepato-Pancreato-Biliary Association Annual Meeting, it was recommended that "postoperative anticoagulant thromboprophylaxis be routinely initiated immediately after hepatectomy in hemodynamically stable patients with no evidence for bleeding and should be continued at least until hospital discharge or return of full mobility.”[13]

While postoperative VTE risk rises with the magnitude of hepatic resection and extends beyond hospital discharge, there are no published data on the use of extended pharmacologic thromboprophylaxis in liver surgery.[12] The purpose of this retrospective study was to assess the safety and efficacy of an extended thromboprophylaxis program following postoperative inpatient thromboprophylaxis in oncologic liver surgery. 


\section{METHODS}

\section{Data Acquisition and Patients}

All patients who underwent hepatectomy (T.A.A.) from August 2013 to April 2016 and were treated with early postoperative and extended chemoprophylaxis were identified from a prospectively maintained liver resection database maintained in the Department of Surgical Oncology at the University of Texas MD Anderson Cancer Center (MDACC).

Preoperative variables included age, sex, American Society of Anesthesiologist (ASA) class, hemoglobin $(\mathrm{g} / \mathrm{dL})$, platelet count $\left(\times 10^{9} / \mathrm{L}\right)$, partial thromboplastin time (PTT, seconds), prothrombin time (PT, seconds), International Normalized Ratio (INR), preoperative chemotherapy, and history of VTE in the past year. Intraoperative variables included extent of hepatectomy, estimated blood loss, intraoperative transfusion, and operative time. As defined by The Brisbane 2000 terminology [17], major hepatectomy was defined as nonanatomic resection of 3 or more segments, left hepatectomy, right hepatectomy, extended left hepatectomy, or extended right hepatectomy.[18]

Postoperative variables included symptomatic VTE (deep vein thrombosis [DVT], pulmonary embolism [PE] and/or catheter associated thrombosis), number of intraoperative and postoperative transfusions, major and non-major bleeding events, platelet count nadir, peak PTT, peak PT, peak INR, renal insufficiency/renal failure, respiratory failure, liver failure, bile leak, return to operating room (ROR), cardiac arrest, stroke, coma, myocardial infarction, sepsis/septic shock, pneumonia, pleural effusion, surgical site infection, organ space infection, fascial dehiscence, urinary tract infection, length of stay, 30-day mortality, and 90-day mortality. Postoperative bile leak and liver failure were defined using International Study Group of Liver Surgery standards.[19,20] Major bleeding was defined as a fall in hemoglobin of $2 \mathrm{~g} / \mathrm{dL}$ in 24 hours, bleeding into a major organ or any bleeding that required reintervention; all other bleeding was classified as non-major bleeding. All complications were collected and graded in real-time using the Modified Accordion Grading system $[21,22]$ and reviewed by two surgical staff members. Severe complications were defined as Accordion grade 3 to 5 .

\section{Follow Up and Radiographic VTE Assessment}

After liver surgery, all patients were planned to have follow-up for at least 5-years on a set schedule of imaging, labs, tumor markers and physical exam every 3-4 months for 2 years, then every 6 months for 2 years and then annually. No patients in this study were lost to follow-up and each patient was followed for at least one year. When patients have these visits with local providers clinical notes, labs and imaging were recovered for review. Within 3 months of surgery $90.3 \%$ of patients underwent routine staging/surveillance imaging with a standard contrast-enhanced computed tomography of the chest, abdomen, and pelvis. Asymptomatic VTE was defined as any CT imaging finding of thrombosis of a catheterassociated vessel, pulmonary artery, other thoracic, splanchnic or ileofemoral vein including a specific diagnosis of DVT and/or pulmonary embolus (PE). 


\section{Thromboprophylaxis Protocol}

The extended thromboprophylaxis protocol for liver resection was initiated in August 2013. Preoperative anticoagulation consisted of Thrombo-Embolic Deterrent (TED) hose and sequential compression devices, which were activated prior to anesthesia induction.[23,24] The first dose of anticoagulant VTE prophylaxis was guided by standardized postoperative order sets, which initiated anticoagulant thromboprophylaxis once hemostasis was assured, either in the post-anesthesia care unit or the morning of postoperative day (POD) 1.

All patients in this study were initiated on subcutaneous unfractionated heparin (5000 mg every 8 hours), converted to daily subcutaneous enoxaparin (40 mg) between POD 1 and 3, and continued on enoxaparin for the remainder of the hospitalization. Weight based and renal dosing were utilized when appropriate. Although postoperatively monitored coagulation parameters were variably elevated in proportion to magnitude of hepatectomy, no changes in thromboprophylaxis were made unless there was a clinical indication, such as bleeding. The extended prophylaxis protocol consisted of the following: after minimallyinvasive and/or minor hepatectomies anticoagulant thromboprophylaxis was extended for 14 total days from the day of surgery and after major hepatectomy, in those patients discharged with a drain or with a history of VTE, for a total of 28 days. A schematic diagram of the perioperative protocol is included in Figure 1. For the six patients on therapeutic-dose VTE prophylaxis to treat a recent preoperative VTE, all were reinitiated onto their therapeuticdose regimen at discharge.

\section{Statistical and IRB Considerations}

Continuous variables are reported as a median value and Range $(\mathrm{R}) /$ interquartile range (IQR) or mean with a Standard Deviation (SD). With 124 patients in this sample, the study is powered in chi-squared analysis at $74 \%$ to detect a statistically significant difference in postoperative VTE rates from $2.9 \%$ (national average) to $0 \%$ (current study) with an alpha error rate of 0.10. Statistical analyses were performed using SPSS Statistics 21 (IBM, Armonk, NY). Analyses of these data was approved by our Institutional Review Board.

\section{RESULTS}

One hundred and twenty-four patients were included in this study. The median age was 57 years (IQR 47.2-65.6 years), 64 (51.6\%) were male sex, and 118 (95.2\%) had an ASA 23. All procedures were initiated for malignant indications (Colorectal Liver Metastasis: 51.6\%, Cholangiocarcinoma: 14.5\%, Hepatocellular Carcinoma: 8.1\%, Other Malignancy: 25.8\%), with one $(0.08 \%)$ patient's pathology returning as a benign liver cyst. Preoperative chemotherapy was received by $72(58.1 \%)$ patients. The median preoperative hemoglobin was $12.8 \mathrm{~g} / \mathrm{dL}$ (IQR 11.6-13.7). The median preoperative platelet count was $203 \times 10^{9} / \mathrm{L}$ (IQR $162-248.5 \times 10^{9} / \mathrm{L}$ ). Additionally, median preoperative PTT, PT, and INR were 29.6 seconds (Range 13.0-49.3 seconds), 13.2 seconds (Range 11.8-16.7 seconds), and 1.0 (Range 0.9-1.35), respectively. Thirty-seven (29.8\%) patients underwent a minimally invasive approach to hepatectomy, 39 (31.5\%) patients underwent major hepatectomy and 16 (12.9\%) patients had a multivisceral resection (Table 1). Pharmacologic thromboprophylaxis was started on postoperative day (POD) 0 for 40 (32.3\%) patients and POD1 for 84 (67.7\%) 
patients. 114 (91.9\%) patients were discharged home on the study's extended pharmacologic thromboprohylaxis protocol. For the remainder, severe thrombocytopenia $(0.8 \%)$, postoperative slowly declining hemoglobin/hematoma $(6.5 \%)$ or other signs of minor bleeding $(2.4 \%)$ were reasons why patients were not discharged to home on extended pharmacologic thromboprophylaxis. All patients were included in the analyses of outcomes.

Overall median operative time was 279 minutes (IQR 197-345 minutes). The median estimated blood loss was $150 \mathrm{~mL}$ (IQR 50-250 mL). The intraoperative, postoperative and overall red cell transfusion rates were $5.6 \%, 8.1 \%$ and $10.5 \%$, respectively. No other blood products were administered. The postoperative median peak PTT, PT, and INR were 32.2 seconds (Range 24.0-52.3 seconds), 15.7 seconds (Range 12.5-22.8 seconds), and 1.2 (Range 1.0-2.1), respectively. The median postoperative nadir platelet count was $152 \times$ $10^{9} / \mathrm{L}$ (Range $56-418 \times 10^{9} / \mathrm{L}$ ). Heparin Induced Thrombocytopenia testing was done in selected patients with significant platelet count deltas and was never positive in this cohort. Median length of stay was 5 days (IQR 3-6 days). The severe complication rate was 5.6\%, with one patient requiring return to the $\mathrm{OR}$ for a non-hemorrhagic indication. There were no 90-day mortalities.

During follow-up, which was complete in all patients, there were no symptomatic VTE (Poisson Confidence Interval: 0-3\%) events in this series (Table 2). One hundred and twelve (90.3\%) patients underwent a standard IV CT scan of the chest, abdomen, and pelvis within 90 days of the operation. None of these patients were diagnosed with VTE on these imaging studies, indicating that no patients developed a major "rebound" thrombosis after withdrawal of extended LMWH. Six (4.8\%) patients preoperatively presented on anticoagulant therapy for a previous VTE that was diagnosed greater than 90 days before surgery; in all cases, these patients were discharged on the extended prophylaxis regiment and transitioned back to a therapeutic dose regiment between post-operative day 14-28. None of these 6 patients developed progression of previous thrombosis or new VTE. Although one patient returned to the operating room, the indication was for wound dehiscence, not hemorrhage. Two patients $(1.6 \%)$ did have minor postoperative bleeding leading to discontinuation of pharmacological thromboprophylaxis; in both cases this resulted in bleeding cessation and neither patient required transfusion or re-intervention.

\section{DISCUSSION}

Hepatobiliary surgery presents unique challenges, with a specific need to have effective hemostasis at the operative site while avoiding venous thrombosis at other sites. These challenges are compounded by concomitant physiologic alterations in prothrombotic and antithrombotic coagulation factors. Compelling data now indicate that the majority of these patients are prothrombotic following liver surgery, that the degree of VTE risk is greater as the magnitude of resection increases, and that as many as one-third of events are diagnosed within 30 days of discharge.[12] Combined, these findings suggest the need for meticulous operative techniques, the judicious use of mechanical and pharmacological VTE prophylaxis, and fastidious post-operative management. 
The aims of this study were to determine the safety and efficacy of early initiation combined with extended (post-discharge) pharmacological VTE prophylaxis after liver surgery. It is important to note that the vast majority of liver surgery is performed for malignant indications. Likewise, the liver surgery performed for nonmalignant indications also carries a high risk (Caprini) score. And although this study has limitations regarding a single-center experience and a focus on oncologic liver surgery, this was a continuous series of patients without exclusions and the thromboprophylaxis protocol is identical for both malignant and benign liver surgery indications. Results indicate that, across a broad spectrum of liver procedures on a total of one hundred and twenty-four consecutive patients, no VTE or major bleeding events occurred during the study period, and the rate of non-major bleeding associated with pharmacological prophylaxis was only $1.6 \%$. These results were validated with both rigorous real-time collection of symptomatic events, as well as radiologic assessment available in $90 \%$ of study patients within 90 days of surgery. Although these follow up imaging studies were not dedicated to the detection of VTE, and may have missed infrainguinal lower extremity DVT and/or small PE, these studies were able to rule-out major central thrombotic events. Such radiologic follow-up to identify asymptomatic events is rare in the postoperative VTE literature.[25-28] Of note, among six patients who presented preoperatively on therapy for previous VTE, none experienced a symptomatic progression of thrombosis and 5 out of 6 patients had follow-up with high quality IV contrast CT imaging that demonstrated improved $(3,50 \%)$ or resolved $(2,33 \%)$ thrombus.

Regarding immediate preoperative dosing, the American College of Chest Physician guidelines [29] describe a unique exclusion for preoperative dosing in procedures with extreme bleeding/transfusion risk, specifically commenting on hepatopancreaticobiliary surgery. Although our institution has reported a low rate of bleeding complications, [30] it is important to note that, nationally, 33\% of liver resection patients are transfused.[2] For this reason, the published guideline for VTE prophylaxis in liver surgery indicates that preoperative dosing is optional.[13] Currently, in our practice, a preoperative dose is given when the indicated operation is a minor hepatectomy or a major hepatectomy with low $a$ priori risk of bleeding. Otherwise, patients systematically start chemical thromboprophylaxis when hemostasis is assured, usually within a few hours of procedure completion (Figure 1).

The safety of any post-operative VTE prophylaxis regimen must be measured by postoperative bleeding events, and particularly bleeding events requiring re-intervention or red blood cell transfusion. Using a consistent strategy starting within one day of surgery and continuing for 2-4 weeks after surgery, the postoperative minor bleeding rates and postoperative transfusion rates were low (1.6\% and $8 \%$, respectively) and there were no patients who required re-intervention for bleeding. Of the two postoperative bleeding events that were identified, neither occurred during the outpatient (extended) portion of the prophylaxis strategy. Combined these data indicate that this strategy is safe. Advanced diagnostic technologies, such as thromboelastography, may further widen the therapeutic index of anticoagulant thromboprophylaxis.[16]

Based on these data, our practice has now broadly adopted extended pharmacological VTE prophylaxis as routine. Since adoption, our internal data reflect a less than $1 \%$ rate of in and outpatient postoperative VTE events and our recent risk-adjusted ACS-NSQIP hepatectomy 
data indicate that we are a low statistical outlier for VTE after hepatectomy. We attribute these data, (obtained in a cancer population at the highest risk for postoperative VTE events) to the perioperative, postoperative, and now post-discharge VTE prophylaxis regimen described in this report (Figure 1).

Historically, in hepatic surgery practice, VTE prophylaxis was avoided all together or started several days after surgery when the INR had crested and started to decline. In contrast, this study reports a modern experience with a practice that initiated thromboprophylaxis much earlier than this historical norm. The VTE prophylaxis regimen used within this study is similar to the one reported by a collaboration between Duke University and University of Pittsburgh. Although they did not practice routine use of post-discharge anticoagulant thromboprophylaxis, they observed a reduction of post-hepatectomy VTE with the utilization of postoperative anticoagulant thromboprophylaxis compared to mechanical prophylaxis alone in liver surgery patients (2.2\% vs $6.3 \%$, respectively, $p=0.03)$.[31] Similar to the findings reported in this study and others, [16] our results reaffirm the safety and suggest the efficacy of early postoperative initiation of pharmacologic thromboprophylaxis in liver patients. Although a small sample size, the systematic addition of extended outpatient post-discharge thromboprophylaxis to a high-risk VTE population was associated with a zero-event rate.

Clinical practice guidelines for VTE prophylaxis are variable. Although not specific to liver surgery, surgeons in the United Kingdom follow the National Institute for Health and Clinical Excellence (NICE) practice guidelines which recommend the use of mechanical thromboprophylaxis with graduated compression stocking or intermittent compression devices in gastrointestinal surgery patients who have an increased risk for VTE.[32] Additionally, the NICE guidelines recommend administration of LMWH or low dose unfractionated heparin in patients at low risk of major bleeding. Lastly, both the American Society of Clinical Oncology and the National Comprehensive Cancer Network recommend that patients with major abdominal/pelvic cancer surgery receive pharmacological VTE prophylaxis for 28 postoperative days.[33,34]

Although there are randomized control trials results supporting the use of pharmacologic thromboprophylaxis in general surgery patients, $[35,36]$ there is a paucity of randomized control trials for pharmacologic thromboprophylaxis after liver resection. This preliminary observational study highlights the safety and potential effectiveness of extended anticoagulation after hepatectomy. To replicate these results, a multi-institution randomized study should be conducted to confirm that extended pharmacological thromboprophylaxis beyond hospital discharge significantly reduces post-hepatectomy VTE.

\section{Acknowledgments}

B. J. Kim is supported by National Institutes of Health grant T32CA009599. These data were presented at the 2017 Annual Meeting of the Americas Hepato-Pancreato-biliary Association 


\section{References}

1. Aloia TA, Fahy BN, Fischer CP, Jones SL, Duchini A, Galati J, Gaber AO, Ghobrial RM, Bass BL. Predicting poor outcome following hepatectomy: analysis of 2313 hepatectomies in the NSQIP database. HPB (Oxford). 2009; 11:510-515. [PubMed: 19816616]

2. Tzeng CW, Cooper AB, Vauthey JN, Curley SA, Aloia TA. Predictors of morbidity and mortality after hepatectomy in elderly patients: analysis of 7621 NSQIP patients. HPB (Oxford). 2014; 16:459-468. [PubMed: 24033514]

3. Adams RB, Aloia TA, Loyer E, Pawlik TM, Taouli B, Vauthey JN. Americas Hepato-PancreatoBiliary A, Society of Surgical O, Society for Surgery of the Alimentary T. Selection for hepatic resection of colorectal liver metastases: expert consensus statement. HPB (Oxford). 2013; 15:91103. [PubMed: 23297719]

4. Madoff DC, Abdalla EK, Gupta S, Wu TT, Morris JS, Denys A, Wallace MJ, Morello FA Jr, Ahrar K, Murthy R, Lunagomez S, Hicks ME, Vauthey JN. Transhepatic ipsilateral right portal vein embolization extended to segment IV: improving hypertrophy and resection outcomes with spherical particles and coils. J Vasc Interv Radiol. 2005; 16:215-225. [PubMed: 15713922]

5. Kishi Y, Zorzi D, Contreras CM, Maru DM, Kopetz S, Ribero D, Motta M, Ravarino N, Risio M, Curley SA, Abdalla EK, Capussotti L, Vauthey J-N. Extended Preoperative Chemotherapy Does Not Improve Pathologic Response and Increases Postoperative Liver Insufficiency After Hepatic Resection for Colorectal Liver Metastases. Annals of Surgical Oncology. 2010; 17:2870-2876. [PubMed: 20567921]

6. Aloia TA, Zorzi D, Abdalla EK, Vauthey J-N. Two-Surgeon Technique for Hepatic Parenchymal Transection of the Noncirrhotic Liver Using Saline-Linked Cautery and Ultrasonic Dissection. Annals of Surgery. 2005; 242:172-177. [PubMed: 16041206]

7. Gold JS, Are C, Kornprat P, Jarnagin WR, Gönen M, Fong Y, DeMatteo RP, Blumgart LH, D’Angelica M. Increased Use of Parenchymal-Sparing Surgery for Bilateral Liver Metastases From Colorectal Cancer Is Associated With Improved Mortality Without Change in Oncologic Outcome: Trends in Treatment Over Time in 440 Patients. Annals of Surgery. 2008; 247:109-117. [PubMed: 18156930]

8. Karanjia ND, Lordan JT, Quiney N, Fawcett WJ, Worthington TR, Remington J. A comparison of right and extended right hepatectomy with all other hepatic resections for colorectal liver metastases: a ten-year study. Eur J Surg Oncol. 2009; 35:65-70. [PubMed: 18222623]

9. Redaelli CA, Wagner M, Krahenbuhl L, Gloor B, Schilling MK, Dufour JF, Buchler MW. Liver surgery in the era of tissue-preserving resections: early and late outcome in patients with primary and secondary hepatic tumors. World J Surg. 2002; 26:1126-1132. [PubMed: 12209242]

10. Sarpel U, Bonavia AS, Grucela A, Roayaie S, Schwartz ME, Labow DM. Does Anatomic Versus Nonanatomic Resection Affect Recurrence and Survival in Patients Undergoing Surgery for Colorectal Liver Metastasis? Annals of Surgical Oncology. 2009; 16:379-384. [PubMed: 19020941]

11. Tzeng CW, Katz MH, Fleming JB, Pisters PW, Lee JE, Abdalla EK, Curley SA, Vauthey JN, Aloia TA. Risk of venous thromboembolism outweighs post-hepatectomy bleeding complications: analysis of 5651 National Surgical Quality Improvement Program patients. HPB (Oxford). 2012; 14:506-513. [PubMed: 22762398]

12. Tzeng CW, Curley SA, Vauthey JN, Aloia TA. Distinct predictors of pre- versus post-discharge venous thromboembolism after hepatectomy: analysis of 7621 NSQIP patients. HPB (Oxford). 2013; 15:773-780. [PubMed: 23869577]

13. Aloia TA, Geerts WH, Clary BM, Day RW, Hemming AW, D’Albuquerque LC, Vollmer CM Jr, Vauthey JN, Toogood GJ. Venous Thromboembolism Prophylaxis in Liver Surgery. J Gastrointest Surg. 2016; 20:221-229. [PubMed: 26489742]

14. Bezeaud A, Denninger MH, Dondero F, Saada V, Venisse L, Huisse MG, Belghiti J, Claude Guillin M. Hypercoagulability after partial liver resection. Thrombosis and Haemostasis. 2007

15. Mukherjee D, Lidor AO, Chu KM, Gearhart SL, Haut ER, Chang DC. Postoperative venous thromboembolism rates vary significantly after different types of major abdominal operations. J Gastrointest Surg. 2008; 12:2015-2022. [PubMed: 18668299] 
16. Le AT, Harris JW, Maynard E, Dineen SP, Tzeng CD. Thromboelastography demonstrates perioperative hypercoagulability in hepato-pancreato-biliary patients and supports routine administration of preoperative and early postoperative venous thromboembolism chemoprophylaxis. HPB (Oxford). 2017; 19:154-161. [PubMed: 27894845]

17. Strasberg SM. Nomenclature of hepatic anatomy and resections: a review of the Brisbane 2000 system. Journal of Hepato-Biliary-Pancreatic Surgery. 2005; 12:351-355. [PubMed: 16258801]

18. Andreou A, Vauthey JN, Cherqui D, Zimmitti G, Ribero D, Truty MJ, Wei SH, Curley SA, Laurent A, Poon RT, Belghiti J, Nagorney DM, Aloia TA. International Cooperative Study Group on Hepatocellular C. Improved long-term survival after major resection for hepatocellular carcinoma: a multicenter analysis based on a new definition of major hepatectomy. J Gastrointest Surg. 2013; 17:66-77. discussion p 77. [PubMed: 22948836]

19. Brooke-Smith M, Figueras J, Ullah S, Rees M, Vauthey JN, Hugh TJ, Garden OJ, Fan ST, Crawford M, Makuuchi M, Yokoyama Y, Buchler M, Weitz J, Padbury R. Prospective evaluation of the International Study Group for Liver Surgery definition of bile leak after a liver resection and the role of routine operative drainage: an international multicentre study. HPB (Oxford). 2015; 17:46-51. [PubMed: 25059275]

20. Rahbari NN, Garden OJ, Padbury R, Brooke-Smith M, Crawford M, Adam R, Koch M, Makuuchi M, Dematteo RP, Christophi C, Banting S, Usatoff V, Nagino M, Maddern G, Hugh TJ, Vauthey JN, Greig P, Rees M, Yokoyama Y, Fan ST, Nimura Y, Figueras J, Capussotti L, Buchler MW, Weitz J. Posthepatectomy liver failure: a definition and grading by the International Study Group of Liver Surgery (ISGLS). Surgery. 2011; 149:713-724. [PubMed: 21236455]

21. Porembka MR, Hall BL, Hirbe M, Strasberg SM. Quantitative weighting of postoperative complications based on the accordion severity grading system: demonstration of potential impact using the american college of surgeons national surgical quality improvement program. J Am Coll Surg. 2010; 210:286-298. [PubMed: 20193891]

22. Strasberg SM, Hall BL. Postoperative morbidity index: a quantitative measure of severity of postoperative complications. J Am Coll Surg. 2011; 213:616-626. [PubMed: 21871822]

23. Nathan H, Weiss MJ, Soff GA, Stempel M, Dematteo RP, Allen PJ, Kingham TP, Fong Y, Jarnagin WR, D’Angelica MI. Pharmacologic prophylaxis, postoperative INR, and risk of venous thromboembolism after hepatectomy. J Gastrointest Surg. 2014; 18:295-302. discussion 302-293. [PubMed: 24129829]

24. Weiss MJ, Kim Y, Ejaz A, Spolverato G, Haut ER, Hirose K, Wolfgang CL, Choti MA, Pawlik TM. Venous thromboembolic prophylaxis after a hepatic resection: patterns of care among liver surgeons. HPB (Oxford). 2014; 16:892-898. [PubMed: 24888461]

25. ENOXACAN Study Group. Efficacy and safety of enoxaparin versus unfractionated heparin for prevention of deep vein thrombosis in elective cancer surgery: a double-blind randomized multicentre trial with venographic assessment. ENOXACAN Study Group. Br J Surg. 1997; 84:1099-1103. [PubMed: 9278651]

26. Harenberg J, Roebruck P, Heene DL. Subcutaneous low-molecular-weight heparin versus standard heparin and the prevention of thromboembolism in medical inpatients. The Heparin Study in Internal Medicine Group. Haemostasis. 1996; 26:127-139. [PubMed: 8738587]

27. McLeod RS, Geerts WH, Sniderman KW, Greenwood C, Gregoire RC, Taylor BM, Silverman RE, Atkinson KG, Burnstein M, Marshall JC, Burul CJ, Anderson DR, Ross T, Wilson SR, Barton P. Canadian Colorectal Surgery DVTPTi. Subcutaneous heparin versus low-molecular-weight heparin as thromboprophylaxis in patients undergoing colorectal surgery: results of the canadian colorectal DVT prophylaxis trial: a randomized, double-blind trial. Ann Surg. 2001; 233:438-444. [PubMed: 11224634]

28. Akl EA, Terrenato I, Barba M, Sperati F, Sempos EV, Muti P, Cook DJ, Schunemann HJ. Lowmolecular-weight heparin vs unfractionated heparin for perioperative thromboprophylaxis in patients with cancer: a systematic review and meta-analysis. Arch Intern Med. 2008; 168:12611269. [PubMed: 18574082]

29. Gould MK, Garcia DA, Wren SM, Karanicolas PJ, Arcelus JI, Heit JA, Samama CM. American College of Chest P. Prevention of VTE in nonorthopedic surgical patients: Antithrombotic Therapy and Prevention of Thrombosis, 9th ed: American College of Chest Physicians Evidence-Based Clinical Practice Guidelines. Chest. 2012; 141:e227S-277S. [PubMed: 22315263] 
30. Day RW, Brudvik KW, Vauthey JN, Conrad C, Gottumukkala V, Chun YS, Katz MH, Fleming JB, Lee JE, Aloia TA. Advances in hepatectomy technique: Toward zero transfusions in the modern era of liver surgery. Surgery. 2016; 159:793-801. [PubMed: 26584854]

31. Reddy SK, Turley RS, Barbas AS, Steel JL, Tsung A, Marsh JW, Clary BM, Geller DA. Postoperative pharmacologic thromboprophylaxis after major hepatectomy: does peripheral venous thromboembolism prevention outweigh bleeding risks? J Gastrointest Surg. 2011; 15:1602-1610. [PubMed: 21691924]

32. Venous Thromboembolism: Reducing the Risk of Venous Thromboembolism (Deep Vein Thrombosis and Pulmonary Embolism) in Patients Admitted to Hospital. London: 2010.

33. Lyman GH, Bohlke K, Falanga A. American Society of Clinical O. Venous thromboembolism prophylaxis and treatment in patients with cancer: American Society of Clinical Oncology clinical practice guideline update. J Oncol Pract. 2015; 11:e442-444. [PubMed: 25873061]

34. Streiff MB, Holmstrom B, Ashrani A, Bockenstedt PL, Chesney C, Eby C, Fanikos J, Fenninger RB, Fogerty AE, Gao S, Goldhaber SZ, Hendrie P, Kuderer N, Lee A, Lee JT, Lovrincevic M, Millenson MM, Neff AT, Ortel TL, Paschal R, Shattil S, Siddiqi T, Smock KJ, Soff G, Wang TF, Yee GC, Zakarija A, McMillian N, Engh AM. Cancer-Associated Venous Thromboembolic Disease, Version 1. 2015. J Natl Compr Canc Netw. 2015; 13:1079-1095. [PubMed: 26358792]

35. Collins R, Scrimgeour A, Yusuf S, Peto R. Reduction in fatal pulmonary embolism and venous thrombosis by perioperative administration of subcutaneous heparin. Overview of results of randomized trials in general, orthopedic, and urologic surgery. N Engl J Med. 1988; 318:11621173. [PubMed: 3283548]

36. Clagett GP, Reisch JS. Prevention of venous thromboembolism in general surgical patients. Results of meta-analysis. Ann Surg. 1988; 208:227-240. [PubMed: 2456748] 


\section{ESSENTIALS}

- The risk for venous thromboembolism after liver surgery remains high in the modern era.

- We evaluated the safety/efficacy of extended anticoagulation in liver surgery.

- This protocol reports zero venous thromboembolism events in 124 liver surgery patients.

- Extended anticoagulation after oncologic liver surgery is safe and effective. 


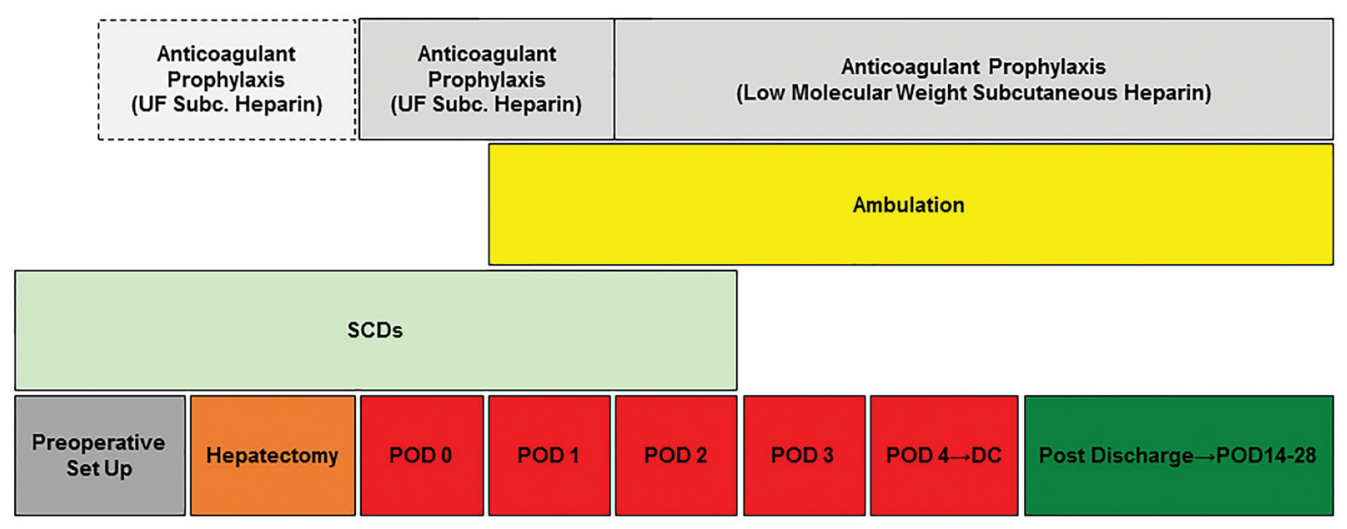

Figure 1.

University of Texas MD Anderson Cancer Center Perioperative Venous Thromboembolism Prophylaxis Protocol in Liver Surgery. Of note, pre-incision dose of pharmacologic prophylaxis (dashed-line box) is variable depending on case-specific bleeding risk. Abbreviations: SCD, Sequential Compression Device; Subc, Subcutaneous; UF, Unfractionated 


\section{Table 1}

Preoperative, Intraoperative, and Postoperative Clinical Characteristics

\begin{tabular}{|c|c|c|}
\hline Clinical Characteristics & n or median & $\%$ or IQR \\
\hline $\mathrm{N}$ & 124 & $100 \%$ \\
\hline \multicolumn{3}{|l|}{ Preoperative } \\
\hline Age, years & 57 & $47.2-65.6$ \\
\hline Sex, male & 64 & $51.6 \%$ \\
\hline $\mathrm{ASA} \geq 3$ & 118 & $95.2 \%$ \\
\hline $\mathrm{Hgb}, \mathrm{g} / \mathrm{dL}$ & 12.8 & $11.6-13.7$ \\
\hline Platelets, median $\times 10^{9} / \mathrm{L}$ & 203 & $162-248.5$ \\
\hline PTT, median secs & 29.6 & $27.6-31.4$ \\
\hline PT, median secs & 13.2 & $12.7-13.7$ \\
\hline INR, median & 1.0 & $0.9-1.0$ \\
\hline Preoperative Chemotherapy & 72 & $58.1 \%$ \\
\hline \multicolumn{3}{|l|}{ Intraoperative } \\
\hline Operative time, mins & 279 & $197-345$ \\
\hline MIS & 37 & $29.8 \%$ \\
\hline Multivisceral Resection & 16 & $12.9 \%$ \\
\hline Major Hepatectomy & 39 & $31.5 \%$ \\
\hline $\mathrm{EBL}, \mathrm{mL}$ & 150 & $50-250$ \\
\hline \multicolumn{3}{|l|}{ Postoperative } \\
\hline Nadir Platelets, median $\times 10^{9} / \mathrm{L}$ & 152 & $123-182$ \\
\hline Peak PTT, median secs & 32.2 & $29.3-36.3$ \\
\hline Peak PT, median secs & 15.7 & $14.8-16.8$ \\
\hline Peak INR, median & 1.2 & $1.1-1.4$ \\
\hline Length of Stay, days & 5 & $3-6$ \\
\hline Any Complication & 22 & $17.7 \%$ \\
\hline Severe Complication & 7 & $5.6 \%$ \\
\hline Liver Failure & 0 & $0.0 \%$ \\
\hline Bile Leak & 1 & $0.8 \%$ \\
\hline Renal Insufficiency/Renal Failure & 1 & $0.8 \%$ \\
\hline Respiratory Failure & 1 & $0.8 \%$ \\
\hline Cardiac arrest & 0 & $0.0 \%$ \\
\hline Stroke & 0 & $0.0 \%$ \\
\hline Myocardial Infarction & 0 & $0.0 \%$ \\
\hline Sepsis/Septic Shock & 0 & $0.0 \%$ \\
\hline Pneumonia & 1 & $0.8 \%$ \\
\hline Pleural Effusion & 2 & $1.6 \%$ \\
\hline Surgical Site Infection & 3 & $2.4 \%$ \\
\hline Wound Dehiscence & 1 & $0.8 \%$ \\
\hline UTI & 4 & $3.2 \%$ \\
\hline Return to operating room & 1 & $0.8 \%$ \\
\hline
\end{tabular}

J Thromb Haemost. Author manuscript; available in PMC 2018 November 01. 


\begin{tabular}{|lcc|}
\hline Clinical Characteristics & n or median & \% or IQR \\
Readmission & 7 & $5.6 \%$ \\
90 Day Mortality & 0 & $0.0 \%$ \\
\hline
\end{tabular}

Abbreviations: ASA, American Society of Anesthesiologist; EBL, Estimated Blood Loss; g/dL, grams/deciliters; Hgb, hemoglobin; INR, International Normalized Ratio; IQR, Interquartile Range; L, Liters; mins, minutes; MIS, Minimally Invasive Surgery; mL, milliliters; OR, Operating Room; PT, prothrombin time; PTT, partial thromboplastin time; secs, seconds; UTI, Urinary Tract Infection. 


\section{Table 2}

Venous Thromboembolism Related Outcomes

\begin{tabular}{|lcc|}
\hline Outcome & Number of Events & $\underline{\text { \% }}$ \\
Bleeding event & 2 & 1.6 \\
Intraoperative Transfusion & 7 & 5.6 \\
Postoperative Transfusion & 10 & 8.1 \\
DVT* $^{*}$ & 0 & 0.0 \\
PE* & 0 & 0.0 \\
\hline
\end{tabular}

Abbreviations: DVT, Deep Vein Thrombosis; PE, Pulmonary Embolism 